\title{
Calculating the Gravitational Self-Force in Schwarzschild Spacetime
}

\author{
Leor Barack, ${ }^{1}$ Yasushi Mino, ${ }^{2}$ Hiroyuki Nakano, ${ }^{3}$ Amos Ori, ${ }^{4}$ and Misao Sasaki ${ }^{3}$ \\ ${ }^{1}$ Albert-Einstein-Institut, Max-Planck-Institut für Gravitationsphysik, Am Mühlenberg 1, D-14476 Golm, Germany \\ ${ }^{2}$ Theoretical Astrophysics, California Institute of Technology, Pasadena, California 91125 \\ ${ }^{3}$ Department of Earth and Space Science, Graduate School of Science, Osaka University, Toyonaka, Osaka 560-0043, Japan \\ ${ }^{4}$ Department of Physics, Technion-Israel Institute of Technology, Haifa 32000, Israel
}

(Received 1 November 2001; published 19 February 2002)

\begin{abstract}
We present a practical method for calculating the local gravitational self-force (often called "radiationreaction force") for a pointlike particle orbiting a Schwarzschild black hole. This is an implementation of the method of mode-sum regularization, in which one first calculates the (finite) contribution to the force due to each individual multipole mode of the perturbation, and then applies a certain regularization procedure to the mode sum. Here we give the values of all the "regularization parameters" required for implementing this regularization procedure, for any geodesic orbit in Schwarzschild spacetime.
\end{abstract}

DOI: $10.1103 /$ PhysRevLett.88.091101

The problem of determining the self-force (SF) acting on a charged particle dates many decades back to the classical works by Lorentz [1] and Dirac [2] on the electron's equation of motion. This force - often referred to as "radiation-reaction force"-may be attributed to the flux of energy momentum carried away by the electromagnetic radiation. The recent years' growing interest in sources of gravitational waves has renewed interest in this old problem, in a new and exciting context: the gravitational SF acting on a point mass in curved spacetime.

This manuscript deals with the motion of a small pointlike particle of mass $\mu$ around a Schwarzschild black hole with mass $M \gg \mu$. When $\mu$ is finite, the particle's trajectory deviates from a geodesic of the Schwarzschild background geometry, due to the particle's interaction with its own gravitational field. This deviation indicates the presence of a self-force. The particle's equation of motion thus takes the form $\mu a_{\alpha}=F_{\alpha}^{\text {self }}$, where $a_{\alpha}$ is the covariant acceleration (i.e., $\left.a_{\alpha}=u_{\alpha ; \beta} u^{\beta}\right)$, and $F_{\alpha}^{\text {self }} \propto O\left(\mu^{2}\right)$ describes the leading-order gravitational SF effect. In general, knowledge of $F_{\alpha}^{\text {self }}$ is essential for modeling the orbital evolution in binary black-hole systems with extreme mass ratios. Such astrophysical systems - typically, the scenario of a small compact object captured by a supermassive black hole (of the kind now believed to reside in the cores of many galaxies, including our own) - are expected to serve as main targets for future space-based gravitational wave detectors [3]. The need for precisely predicting the orbital evolution of such binary systems, and the waveform of the emitted gravitational radiation, strongly motivates the development of practical methods for gravitational SF calculations.

When dealing with point particles, one faces the fundamental issue of regularization: extracting the "correct" finite part of the (otherwise divergent) SF. A formalism for regularizing the electromagnetic SF in curved spacetime was developed long ago by DeWitt and Brehme [4]. Recently, Mino, Sasaki, and Tanaka (MST) [5] first obtained a formal expression for the gravitational SF, based on a
PACS numbers: 04.70.Bw, 04.25.Nx

physically well-motivated regularization technique. The same formal result was independently derived by Quinn and Wald (QW) [6] using an axiomatic approach. It is useful to write this result as

$$
F_{\alpha}^{\text {self }}=\lim _{x \rightarrow z_{0}}\left[F_{\alpha}^{\text {full }}(x)-F_{\alpha}^{\mathrm{dir}}(x)\right]
$$

where $z_{0}^{\mu}$ is the SF's evaluation point, $x^{\mu}$ is a point in the neighborhood of $z_{0}^{\mu}, F_{\alpha}^{\text {full }}$ is the "full" force constructed from the metric perturbation (as described below), and $F_{\alpha}^{\mathrm{dir}}$ (the "direct" force) is the "divergent piece" to be removed, whose formal construction is described in $[5,6]$. Roughly speaking, the direct piece $F_{\text {dir }}^{\alpha}$ represents the instantaneous effect of waves propagating directly along the particle's light cone; hence, the SF effect is entirely attributed to the so-called "tail" part of the particle's field, i.e., waves scattered inside the particle's past light cone due to the background's curvature.

The above regularization prescription by MST is based on the physically well-motivated method of matched asymptotic expansions. At present, this prescription, which also conforms with QW's prescription, is widely accepted as the standard regularization scheme for the SF [7]. As it stands, however, it is difficult to directly implement this method in practical calculations, because it requires the computation of the Green's function from any world line point to any other future world line point [8]. To allow the practical implementation of Eq. (1) in strong-field calculations, Barack and Ori (BO) devised a method based on a mode decomposition of the perturbation field (see [9] for the scalar SF and [10] for the gravitational SF). In the mode-sum method, the quantities $F_{\alpha}^{\text {full }}$ and $F_{\alpha}^{\mathrm{dir}}$ are first formally expanded into multipole $l$ modes $F_{\alpha l}^{\mathrm{full}}$ and $F_{\alpha l}^{\mathrm{dir}}$. Most beneficial is the fact that, whereas $F_{\alpha}^{\text {full }}$ diverges as $x \rightarrow z_{0}$, its individual modes attain finite values even at the particle's location. The full mode $F_{\alpha l}^{\text {full }}$ is directly derived (see below) from the $l$-mode perturbation field, which, in turn, is obtained from the 
decoupled field equations $[10,11]$ by standard numerical techniques. (The construction of $F_{\alpha l}^{\mathrm{full}}$ involves summation over the azimuthal number $m$, and, in the gravitational SF case, also over the ten tensorial harmonics for each multipole number $l$.) The direct piece $F_{\alpha}^{\text {dir }}$ (and, hence, its $l$ mode $F_{\alpha l}^{\mathrm{dir}}$ ) is analyzed by local methods (see below). The expression (1) for the SF is then brought to the form $[9,10]$

$$
F_{\alpha}^{\text {self }}=\sum_{l=0}^{\infty}\left[\lim _{x \rightarrow z_{0}} F_{\alpha l}^{\text {full }}-A_{\alpha} L-B_{\alpha}-C_{\alpha} / L\right]-D_{\alpha},
$$

where hereafter the limit $x \rightarrow z_{0}$ is taken along the (ingoing or outgoing) radial direction, $L \equiv l+1 / 2$, and $A_{\alpha}$, $B_{\alpha}, C_{\alpha}$, and $D_{\alpha}$ are certain "regularization parameters" (RP), derived from the local structure of $F_{\alpha l}^{\mathrm{dir}}$ : Roughly speaking, $A_{\alpha} L+B_{\alpha}+C_{\alpha} / L$ reflects the asymptotic form of $F_{\alpha l}^{\mathrm{dir}}$ (and also $F_{\alpha l}^{\text {full }}$ ) at large $l$, while $D_{\alpha}$ is a residual quantity that arises in the summation over $l$ (see $[9,10]$ for a more precise definition of the RP). Note that in the framework of the mode-sum method the task of calculating the SF is divided into two separate parts: (i) calculating the quantities $F_{\alpha l}^{\text {full }}$, e.g., by numerically integrating the decomposed field equations; (ii) analytically calculating the four RP, by locally analyzing the direct part [12]. It is the second part, the analytic derivation of the RP, that will primarily concern us in this Letter.

In the original method by $\mathrm{BO}$, the analysis of $F_{\alpha l}^{\mathrm{dir}}$ was carried out by applying a systematic perturbation expansion of the ( $l$-mode) Green's equation. The derivation of the full set of four RP required the calculation of the Green function up to second order in this expansion. So far, the $\mathrm{RP}$ were calculated using this original method in a few special cases: the scalar SF for a particle in radial or circular orbits [9], and the gravitational SF for radial motion $[10,13]$ (all in Schwarzschild spacetime). However, the required second-order perturbation analysis of the Green function turned out to be rather cumbersome, making it difficult to extend the calculation to more general cases.

The goal of this Letter is to report on a new method for analytically deriving the four RP, which proves to be significantly simpler than the original BO's method. This new mode-sum method is based on an analytic expression constructed by Mino, Nakano, and Sasaki (MNS) [14,15] for the direct force $F_{\alpha}^{\mathrm{dir}}$. (MNS developed this expression as part of a more comprehensive study aimed to provide a systematic description of the local structure of the metric perturbation.) Once this expression is available, essentially all we need is to decompose it into $l$ modes, and then take the limit $x \rightarrow z_{0}$. The large- $l$ asymptotic behavior of this expansion turns out to be a power series in $1 / L$, starting at $L^{1}$, and its three leading-order coefficients yield the three RP $A_{\alpha}, B_{\alpha}$, and $C_{\alpha}$ according to

$$
\lim _{x \rightarrow z_{0}} F_{\alpha l}^{\mathrm{dir}}=A_{\alpha} L+B_{\alpha}+C_{\alpha} / L+O\left(L^{-2}\right) .
$$

The last parameter $D_{\alpha}$ is then obtained by

$$
D_{\alpha}=\sum_{l=0}^{\infty}\left[\lim _{x \rightarrow z_{0}} F_{\alpha l}^{\mathrm{dir}}-A_{\alpha} L-B_{\alpha}-C_{\alpha} / L\right] .
$$

Decomposing the right-hand side of Eq. (1) and then substituting Eq. (4), one recovers Eq. (2). (Note that, since the tail part is regular, $F_{\alpha l}^{\mathrm{full}}$ and $F_{\alpha l}^{\mathrm{dir}}$ admit the same large- $l$ asymptotic behavior, with the same coefficients.)

Applying this new method, we have been able to derive the values of all $\mathrm{RP}$ for an arbitrary geodesic orbit in Schwarzschild spacetime, for both the scalar and the gravitational self-force, as we now describe. Throughout this paper we use geometrized units $G=c=1$ and metric signature -+++ , and denote the standard Schwarzschild coordinates by $t, r, \theta$, and $\varphi$.

We begin by considering a simple scalar-field toy model. Once the scalar case is well understood, it becomes fairly straightforward to obtain the RP in the gravitational case as well. We shall consider a particle carrying a scalar charge $q$, which (in the lack of SF) moves along an equatorial geodesic $z^{\mu}(\tau)$ of the Schwarzschild background ( $\tau$ being the proper time) with specific energy and angular momentum $\mathcal{E}$ and $\mathcal{L}$, respectively. The scalar field $\Phi$ satisfies the field equation $\Phi_{; \alpha}^{; \alpha}=-4 \pi \rho$, where $\rho=q \int \delta^{4}(x-z)(-g)^{-1 / 2} d \tau$ is the scalar charge density ( $g$ being the metric determinant). In this model, the force exerted on the scalar charge by a (external, regular) scalar field $\Phi(x)$ is simply given by $F_{\alpha}=q \Phi_{, \alpha}$.

Let $\epsilon(x)$ denote the spatial distance from $x^{\mu}$ to the world line $z^{\mu}(\tau)$ (i.e., the length of the short geodesic connecting $x^{\mu}$ to the world line and normal to it). Using the Hadamard expansion of the field equation, MNS have been able to show that the direct scalar force takes the form [15]

$$
F_{\alpha}^{\mathrm{dir}}(x)=q \Phi_{, \alpha}^{\mathrm{dir}}, \quad \Phi^{\mathrm{dir}}(x) \equiv q \hat{f} / \epsilon,
$$

where $\Phi^{\operatorname{dir}}(x)$ is the so-called "direct scalar field", and $\hat{f}(x)$ is some regular scalar function satisfying $\hat{f}=1+$ $O\left(\delta x^{2}\right)$, where $\delta x^{\mu} \equiv x^{\mu}-z_{0}^{\mu}$ (the explicit form of $\hat{f}$ will not be needed here). For the analysis below, it is useful to define $S \equiv \epsilon^{2}$. The direct force then takes the form

$$
F_{\alpha}^{\mathrm{dir}}(x)=q^{2}\left(S^{-1 / 2} \hat{f}_{, \alpha}-\hat{f} S^{-3 / 2} S_{, \alpha} / 2\right) .
$$

Being a regular function of $x, S$ may be expanded around $z_{0}$ as $S=S_{0}+O\left(\delta x^{3}\right)$, where [16] $S_{0}=$ $\left(g_{\mu \nu}+u_{\mu} u_{\nu}\right) \delta x^{\mu} \delta x^{\nu}$, and $u^{\mu} \equiv d z^{\mu} / d \tau$. (In the expression for $S_{0}, g_{\mu \nu}$ and $u_{\alpha}$ are to be evaluated at $z_{0}$.)

In the next step we need to decompose the components $F_{\alpha}^{\text {dir }}$ into spherical harmonics and then derive the four RP, as prescribed above. This goal was undertaken independently (and in two slightly different ways) by our two groups, BO and MNS, as we now describe [17].

In the analysis by BO [16], the expression $S=S_{0}+$ $O\left(\delta x^{3}\right)$ is first substituted in Eq. (6). With some simple manipulations, this equation can be brought to the form

$$
F_{\alpha}^{\mathrm{dir}}(x)=\epsilon_{0}^{-3} P_{\alpha}^{(1)}+\epsilon_{0}^{-5} P_{\alpha}^{(4)}+\epsilon_{0}^{-7} P_{\alpha}^{(7)}+O(\delta x),
$$


where $\epsilon_{0} \equiv S_{0}^{1 / 2}$, and $P_{\alpha}^{(n)}$ denote terms of homogeneous order $n$ in $\delta x^{\alpha}$. Notice that the three terms on the righthand side are of order $\delta x^{-2}, \delta x^{-1}$, and $\delta x^{0}$, respectively. The $O(\delta x)$ correction term will not concern us here, as it does not contribute to the direct force at $z_{0}$.

As explained above, the mode $l$ of $F_{\alpha}^{\mathrm{dir}}$ (or $F_{\alpha}^{\mathrm{full}}$ ) is obtained by summing the contributions of all possible values of $m$ for this specific $l$. This sum is invariant under rotation of the angular variables $\theta, \varphi$. Using this invariance, BO chose a new, rotated, set of angular variables $\theta^{\prime}, \varphi^{\prime}$ (with respect to which the spherical harmonics are to be defined), such that the evaluation point $z_{0}$ is located at the pole, $\theta^{\prime}=0$. Since all $m \neq 0$ spherical harmonics vanish at $\theta^{\prime}=0$, with these rotated coordinates one needs to consider only the $m=0$ modes. This greatly simplifies the analysis. The presence of a coordinate singularity (the trivial $\theta^{\prime}=0$ singularity) at the evaluation point somewhat complicates the situation. To overcome this difficulty, BO introduced two regular "Cartesian-like" coordinates $x, y$. With this choice of coordinates $(t, r, x, y)$, the $l$ decomposition of Eq. (7) becomes especially simple. After taking the limit $x \rightarrow z_{0}$, one finds that (i) the term $\epsilon_{0}^{-7} P_{\alpha}^{(7)}$ yields zero contribution to $F_{\alpha l}^{\mathrm{dir}}$, (ii) the term $\epsilon_{0}^{-5} P_{\alpha}^{(4)}$ yields a constant (i.e., $l$ independent) contribution which we denote $b_{\alpha}$, and (iii) the contribution of the term $\epsilon_{0}^{-3} P_{\alpha}^{(1)}$ is precisely proportional to $L$; we shall denote it $a_{\alpha} L$. From Eqs. (3) and (4) it now follows that $A_{\alpha}=a_{\alpha}, B_{\alpha}=$ $b_{\alpha}$, and $C_{\alpha}=D_{\alpha}=0$. The detailed calculation of the coefficients $a_{\alpha}$ and $b_{\alpha}$ is given in Ref. [16]. The final expressions obtained by BO for the RP (expressed in the original $\theta, \varphi$ coordinates, in which the motion is equatorial) are given by $A_{\theta}^{\mathrm{sc}}=B_{\theta}^{\mathrm{sc}}=0$,

$$
\begin{gathered}
A_{ \pm r}^{\mathrm{sc}}=\mp \frac{q^{2}}{r^{2}} \frac{\mathcal{E}}{f V}, \quad A_{ \pm t}^{\mathrm{sc}}= \pm \frac{q^{2}}{r^{2}} \frac{\dot{r}}{V}, \quad A_{\varphi}^{\mathrm{sc}}=0, \\
B_{r}^{\mathrm{sc}}=\frac{q^{2}}{r^{2}} \frac{\left(\dot{r}^{2}-2 \mathcal{E}^{2}\right) \hat{K}(w)+\left(\dot{r}^{2}+\mathcal{E}^{2}\right) \hat{E}(w)}{\pi f V^{3 / 2}}, \\
B_{t}^{\mathrm{sc}}=\frac{q^{2}}{r^{2}} \frac{\mathcal{E} \dot{r}[\hat{K}(w)-2 \hat{E}(w)]}{\pi V^{3 / 2}} \\
B_{\varphi}^{\mathrm{sc}}=\frac{q^{2}}{r} \frac{\dot{r}[\hat{K}(w)-\hat{E}(w)]}{\pi(\mathcal{L} / r) V^{1 / 2}} \\
C_{\alpha}^{\mathrm{sc}}=D_{\alpha}^{\mathrm{sc}}=0
\end{gathered}
$$

(with "sc" signifying the scalar force RP), where $\hat{K}(w)$ and $\hat{E}(w)$ are the complete elliptic integrals of the first and the second kinds, respectively, $w \equiv \mathcal{L}^{2} /\left(\mathcal{L}^{2}+r^{2}\right), f \equiv$ $(1-2 M / r), V \equiv 1+\mathcal{L}^{2} / r^{2}$, and $\dot{r} \equiv d r / d \tau$. The “ \pm " sign in $A_{\alpha}$ refers to whether the limit $x \rightarrow z_{0}$ is taken along the ingoing or outgoing radial direction. The values (8) agree with those obtained in [9] in the special cases $\mathcal{L}=0$ or $\dot{r}=0$.

In an independent analysis, MNS directly used the "standard" $\theta, \varphi$ coordinates (in which the motion is equatorial) for decomposing the direct field. In this setup, the $m \neq 0$ modes contribute as well. MNS derived an analytic expression for the contribution of each $l, m$ mode of the direct force, expanded in powers of $M / r$. Then, MNS have shown the convergence of this expansion, and were able to explicitly sum it up (and sum over $m$ ), after which they recovered all RP values (8).

We now turn to discuss the gravitational SF. Let $\bar{h}_{\alpha \beta}^{\text {full }} \equiv \bar{h}_{\alpha \beta}$ denote the trace-reversed metric perturbation induced by a particle of mass $\mu$ (namely, $\bar{h}_{\alpha \beta} \equiv h_{\alpha \beta}-\frac{1}{2} g_{\alpha \beta} g^{\mu \nu} h_{\mu \nu}$, where $h_{\alpha \beta}$ is the metric perturbation itself, and $g_{\alpha \beta}$ is the background metric). Just as in the scalar case, this full perturbation is the sum of the direct part and the tail part, $\bar{h}_{\alpha \beta}^{\text {full }}=\bar{h}_{\alpha \beta}^{\text {dir }}+\bar{h}_{\alpha \beta}^{\text {tail }}$. The gravitational SF is then obtained by applying a certain differential operator to $\bar{h}_{\alpha \beta}^{\text {tail }}$ :

$$
F_{\alpha}^{\text {self }}=F_{\alpha}^{\text {tail }} \equiv \mu k_{\alpha}{ }^{\beta \gamma \delta} \bar{h}_{\beta \gamma ; \delta}^{\text {tail }},
$$

evaluated at $x \rightarrow z_{0}$, where

$$
\begin{aligned}
k^{\alpha \beta \gamma \delta}= & u^{\beta} u^{\gamma} g^{\alpha \delta} / 2+g^{\beta \gamma} g^{\alpha \delta} / 4+u^{\alpha} g^{\beta \gamma} u^{\delta} / 4 \\
& -g^{\alpha \beta} u^{\gamma} u^{\delta}-u^{\alpha} u^{\beta} u^{\gamma} u^{\delta} / 2
\end{aligned}
$$

all quantities evaluated at $z_{0}$. Let us also define

$$
F_{\alpha}^{(\mathrm{dir}, \text { full })}=\mu k_{\alpha}{ }^{\beta \gamma \delta} \bar{h}_{\beta \gamma ; \delta}^{(\mathrm{dir}, \mathrm{full})},
$$

after which Eq. (1) is recovered.

The direct trace-reversed metric perturbation was found by MST [5]. It can be expressed as

$$
\bar{h}_{\beta \gamma}^{\mathrm{dir}}(x)=4 \mu \bar{f}(x, z) \epsilon^{-1} \hat{u}_{\beta} \hat{u}_{\gamma},
$$

where $\bar{f}$ is a regular function satisfying $\bar{f}=1+O\left(\delta x^{2}\right)$, and $\hat{u}_{\alpha}$ is the four-velocity parallelly propagated from the particle's world line to $x$ along the short normal geodesic.

As it stands, the tensor $k$ is defined only on the particle's world line, because it involves $u^{\alpha}$. In Eq. (9) this on-world-line definition suffices, because $\bar{h}^{\text {tail }}$ is regular at the particle's location [5]. However, $\bar{h}^{\text {full }}$ and $\bar{h}^{\text {dir }}$ diverge as $1 / \epsilon$ (and their gradients as $\epsilon^{-2}$ ). Therefore, when using Eq. (11) [e.g., when substituting it in Eq. (1)], we must prescribe an extension of $k$ off the world line. Recall, however, that no ambiguity is caused by this nonuniqueness of $k$ : One just needs to use the same extension for both the direct and the full forces. Below we consider several extensions: The one obtained by substituting $u^{\alpha} \rightarrow \hat{u}^{\alpha}$ is denoted $\hat{k}$; and the extension based on "fixed (contravariant) components" (in the Schwarzschild coordinates) is denoted $\tilde{k}$. A third extension, mentioned below, is denoted $\bar{k}$. Correspondingly, we shall denote the quantities associated with the above three extensions (e.g., the RP) by a hat, tilde, or an overbar, respectively.

The gravitational RP are obtained by decomposing the components $F_{\alpha}^{\mathrm{dir}}$ into scalar spherical harmonics, just as in the scalar case. In the $\hat{k}$ extension, MNS obtained [15] $\hat{R}_{\alpha}^{\mathrm{gr}}=R_{\alpha}^{\mathrm{sc}}$ (with $q \rightarrow \mu$ ), where $R_{\alpha}$ stands for all four RP, and the label "gr" signifies the gravitational case values. In the $\tilde{k}$ extension, BO found [16] $\tilde{R}_{\alpha}^{\mathrm{gr}}=K_{\alpha}^{\lambda} R_{\lambda}^{\mathrm{sc}}$, where $K_{\alpha}^{\lambda} \equiv \delta_{\alpha}^{\lambda}+u_{\alpha} u^{\lambda}$.

091101-3 
For implementing the mode-sum method, Eq. (2), we must have at hand $F_{\alpha l}^{\text {full }}$, the spherical harmonic $l$ mode of the ( $\alpha$ component of the) full force. In the scalar case, this quantity is obtained by numerically computing the full-field mode $\Phi_{l}$ and differentiating it with respect to $x^{\alpha}$. In the gravitational case the procedure is a bit more involved. The linearized Einstein equations are separated by the decomposition,

$$
\bar{h}_{\alpha \beta}=\sum_{l m} \sum_{i=1}^{10} \bar{h}^{(i) l m}(r, t) Y_{\alpha \beta}^{(i) l m}(\theta, \varphi),
$$

where $Y_{\alpha \beta}^{(i) l m}$ are the ten (Zerilli-type) tensorial harmonics [10]. The quantities $\bar{h}^{(i) l m}$ can be computed by numerically integrating the decoupled field equations. Then,

$$
F_{\alpha}^{\mathrm{full}}=\mu \sum_{i l m} k_{\alpha}{ }^{\beta \gamma \delta}\left[\bar{h}^{(i) l m}(r, t) Y_{\beta \gamma}^{(i) l m}(\theta, \varphi)\right]_{; \delta} .
$$

To obtain $F_{\alpha l}^{\text {full }}$, we need to decompose this quantity in ordinary (i.e., scalar) spherical harmonics $Y^{l m}(\theta, \varphi)$, and to sum over $i$ and $m$. The outcome of this decomposition will depend on the extension of $k$. This decomposition appears to be difficult to implement with the $\hat{k}$ extension. For most extensions (including $\tilde{k}$ ), an infinite number of tensorial harmonics $\bar{h}^{(i) l^{\prime} m}$ will contribute to a single scalarharmonic term $F_{\alpha l m}^{\text {full }}$ (due to the nontrivial dependence of $k$ on $\theta$ ). However, BO designed a third extension $\bar{k}=$ $\tilde{k}+\delta k$, where $\delta k$ is a certain $O\left(\delta x^{2}\right)$ correction term (see [16]), in which only a finite number $\left(l-3 \leq l^{\prime} \leq l+3\right)$ of tensorial harmonics $\bar{h}^{(i) l^{\prime} m}$ contribute to a single term $F_{\alpha l m}^{\mathrm{full}}$. Then $F_{\alpha l}^{\mathrm{full}}$ is given by

$$
F_{\alpha l}^{\mathrm{full}}=\mu \sum_{i=1}^{10} \sum_{l^{\prime}=l-3}^{l+3} \sum_{m=-l^{\prime}}^{l^{\prime}}\left[\mathcal{D}_{\alpha l}^{(i) l^{\prime} m} \bar{h}^{(i) l^{\prime} m}(r, t)\right] Y^{l m},
$$

where $\mathcal{D}_{\alpha l}^{(i) l^{\prime} m}$ is a certain first-order differential operator (independent of $\theta, \varphi$ ) given explicitly in [16]. It can be shown [16] that the extension difference $\delta k$, being $O\left(\delta x^{2}\right)$, does not modify the RP values, hence,

$$
\bar{R}_{\alpha}^{\mathrm{gr}}=\tilde{R}_{\alpha}^{\mathrm{gr}}=K_{\alpha}^{\lambda} R_{\lambda}^{\mathrm{sc}}
$$

These values coincide, for $\mathcal{L}=0$, with the ones obtained [13] using local analysis of the Green function.

Let us now summarize the prescription for constructing the gravitational SF: (i) Numerically compute the radial functions $\bar{h}^{(i) l^{\prime} m}(r, t)$ (e.g., in the harmonic gauge [10]); (ii) use Eq. (15) to construct the full modes $F_{\alpha l}^{\text {full }}$; (iii) use Eq. (16) [along with Eq. (8), with $q \rightarrow \mu$ ] to obtain the RP; and (iv) apply Eq. (2). This prescription is now being implemented by Barack and Lousto [13].

Finally, it should be reminded that the gravitational SF is a gauge-dependent notion, as discussed in Ref. [18]. Nevertheless, the RP are gauge independent [18]. The above prescription applies to the SF associated with the harmonic gauge, or any other gauge related to it by a regular gauge transformation.

We would like to thank Lior Burko for interesting discussions and stimulating interaction. L. B. was supported by the European Community program IHP-MCIF-99-1 (Marie Curie Fund) under Contract No. HPMF-CT-2000-00851. Y. M. was supported by NSF Grants No. PHY-0099568 and PHY-0096522 and NASA Grant No. NAG5-10707. H. N. and M. S. were supported in part by a Monbusho Grant-in-Aid for Creative Research (No. 09NP0801), and by a Monbusho Grant-in-Aid for Scientific Research (No. 12640269). H. N. was also supported by the Japan Society for the Promotion of Science for Young Scientists, No. 2397.

[1] H. A. Lorentz, Theory of Electrons (Dover, New York, 1952), 2nd ed.

[2] P. A. M. Dirac, Proc. R. Soc. London A 167, 148 (1938).

[3] The NASA-ESA project LISA (Laser Interferometer Space Antenna) is now scheduled to be launched around 2011. See the LISA webpage: http://lisa.jpl.nasa.gov/

[4] B. S. DeWitt and R. W. Brehme, Ann. Phys. (N.Y.) 9, 220 (1960).

[5] Y. Mino, M. Sasaki, and T. Tanaka, Phys. Rev. D 55, 3457 (1997).

[6] T. C. Quinn and R. M. Wald, Phys. Rev. D 56, 3381 (1997); see also T. C. Quinn, ibid. 62, 064029 (2000) for analysis of the scalar SF.

[7] For alternative SF regularization techniques, see, e.g., C. O. Lousto, Phys. Rev. Lett. 84, 5251 (2000); S. Detweiler, ibid. 86, 1931 (2001); H. Nakano and M. Sasaki, Prog. Theor. Phys. 105, 197 (2001).

[8] See, however, a recent implementation of the MST-QW approach for a particle moving in a weak-field spacetime: M. J. Pfenning and E. Poisson, gr-qc/0012057 [Phys. Rev. D. (to be published)].

[9] L. Barack and A. Ori, Phys. Rev. D 61, 061502(R) (2000); L. Barack, ibid. 62, 084027 (2000).

[10] L. Barack, Phys. Rev. D 64, 084021 (2001).

[11] T. Regge and J. A. Wheeler, Phys. Rev. 108, 1063 (1957); F. J. Zerilli, Phys. Rev. D 2, 2141 (1970).

[12] For implementation of this method in the scalar case, including both analytic and numerical parts, see L. M. Burko, Classical Quantum Gravity 17, 227 (2000); Phys. Rev. Lett. 84, 4529 (2000); L. Barack and L. M. Burko, Phys. Rev. D 62, 084040 (2000); L. M. Burko, Y.-T. Liu, and Y. Soen, ibid. 63, 024015 (2001); L. M. Burko and Y.-T. Liu, ibid. 64, 024006 (2001).

[13] L. Barack and C. O. Lousto (unpublished).

[14] Y. Mino and H. Nakano, Prog. Theor. Phys. 100, 507 (1998).

[15] Y. Mino, H. Nakano, and M. Sasaki, gr-qc/0111074 (to be published).

[16] L. Barack and A. Ori (to be published).

[17] The same approach was used independently by Burko (unpublished), who applied it for a radial orbit using numerical decomposition of the direct part derived by MNS.

[18] L. Barack and A. Ori, Phys. Rev. D 64, 124003 (2001). 